\title{
Dengue Reemergence: The Challenges Ahead
}

\author{
Amos Watentena*, Ikem C. Okoye, Ikechukwu E. Onah \\ Department of Zoology and Environmental Biology, Faculty of Biological Sciences, University of Nigeria, \\ Nsukka, Nigeria
}

* Corresponding author email: wateamos@gmail.com

Received: 09 May 2019 / Accepted: 01 June 2020 / Published: 08 June 2020

\begin{abstract}
The global expansion of competent Dengue vectors in tropical and sub-tropical areas, and the favorable conditions for disease transmission demonstrates a critical public health challenge ahead. This review provides an insight into some key underlying gaps while attempting to suggest alternatives towards the containment and hence eradication of Dengue. The non-existence of an efficient vaccine against all DENV serotypes and the rapid global emergence of COVID-19, are undermining the complexities of vaccine development and disrupting global health efforts for the management of Dengue respectively. The development of sustainable DENV control programs now requires a multifaceted approach involving the integration of the recently developed multi-platforms application in entomological surveillance, community awareness, modelling, vaccination, vector control, and also ensuring that Dengue control efforts are not hampered or neglected during COVID-19 pandemic. This approach requires active collaboration and communication of scientists from various disciplines, economic support from funding agencies and institutions as well as support from the communities, governments and public health organizations.
\end{abstract}

Keywords: Dengue, DENV, Aedes aegypti, Aedes albopictus, vaccines, vector control.

\section{Dengue and Dengue Virus}

Dengue fever, also commonly known as Dengue, is a mosquito-borne arboviral infection that has become the most rapidly expanding mosquitoborne infectious disease on Earth, surpassing malaria [1]-[4]. Over the past few decades, the number of people infected with Dengue fever has risen exponentially reaching over 100 million people per annum with 22,000 deaths and placing almost 2.5 billion people at risk [2], [4], [5]. This expansion has been attributed to increased urbanization, global travel and commerce, and paucity of mosquito control programs [5]. Dengue fever or Dengue is caused by an infection with Dengue virus [2]. Dengue virus (DENV) is a mosquito-transmitted single-stranded, positivesense RNA virus that is a member of the genus Flavivirus and cycles in nature between its mosquito vectors and humans [5]. It comprises ten proteins; three proteins, capsid (C), membrane $(\mathrm{M})$, and envelope $(\mathrm{E})$, which play a structural role with the other seven proteins identified as non-structural but direct DENV replication [2]. Four distinct serotypes: DENV-1, DENV-2, DENV-3 and DENV-4 circulate in tropical and sub-tropical regions of the globe with each capable of causing severe disease and are transmitted via Aedes mosquitoes [2], [5]. DENV serotypes differ from one another by $25 \%-40 \%$ at the amino acid level and are separated further into genotypes that vary by up to 3\% [5]. Clinically, Dengue patients tend to exhibit: extreme fatigue; sudden fever (from 3-7 days), headache, joint, muscle, and back pain; vomiting and diarrhea, appetite loss; skin rash along with minor bleeding [2]. There is no effective treatment for Dengue fever other than supportive care especially for severe cases, also called Dengue Hemorrhagic Fever (DHF) and Dengue shock syndrome [4]. 


\section{$2 \quad$ Historical Background}

The word "dengue" is derived from Swahili words "ki denga pepo", which translates "sudden cramp like seizure" [2], [6]. The signs and symptoms which are suggestive of the disease can be traced back as early as 265-420 AD in the Chinese Chin Dynasty where it was believed to be a type of water poison linked to insects and water [2], [6], [7]. An ancient clinical dengue-like illness was also recorded in a Chinese medical encyclopedia in 992 [8]. However, the first reported epidemics of dengue fever occurred in 1779-1780 in Asia, Africa, and North America [7], [9]. In India, the first recorded epidemic of clinically dengue-like illness occurred at Madras in 1780 and later, DENV was isolated for the first time almost simultaneously in Japan and Calcutta in 1943-1944 [8], [10].

\subsection{Dengue in the Asian region}

The major occurrence of events during the expansion of Dengue in Asia is summarized in Figure 1 below. Dengue illness was first virologically proved to be a dengue fever epidemic in around 1963 along the East Coast of India where it had spread throughout the country causing the first full-blown epidemic of dengue hemorrhagic fever/dengue shock syndrome in North India in 1996 [8].

Whereas dengue had been eradicated from China for about 32 years, the 1978 epidemic and several other localized epidemics in the 1980s attributed to Aedes aegypti vector in coastal areas and Aedes albopictus vector in inland regions, suggested that the reemergence of dengue in China resulted from the introduction of the infection by travelers and refugees from areas of Asia where dengue is endemic [9].

\subsection{Dengue in the African Region}

Epidemic dengue fever caused by all four serotypes started increasing dramatically in Africa in 1980s, particularly in the parts of Kenya, Somalia and Mozambique, with epidemics most attributed to DENV-2 \& 3 [2], [12]. Dengue epidemics caused by DENV-1 \& 2, have also long been reported in parts of West Africa (particularly Nigeria) although there is scanty information on DHF because of its overlap in clinical symptoms with Dengue fever [13], [14]. Sporadic cases clinically compatible with DHF have also been reported from Mozambique and Djibouti [12]. Dengue outbreaks and epidemics have been reported in all regions of Africa, and it is believed that all four DENV serotypes are in circulation although available data suggests that it is endemic to 34 African countries [15].

Current dengue prevention strategies in Africa focus on vector control, although the primary aim of such efforts is typically the prevention of malaria. Further research is needed to characterize the epidemiology of dengue in Africa and to better understand the factors involved in differences in vulnerability to dengue across Africa [15]

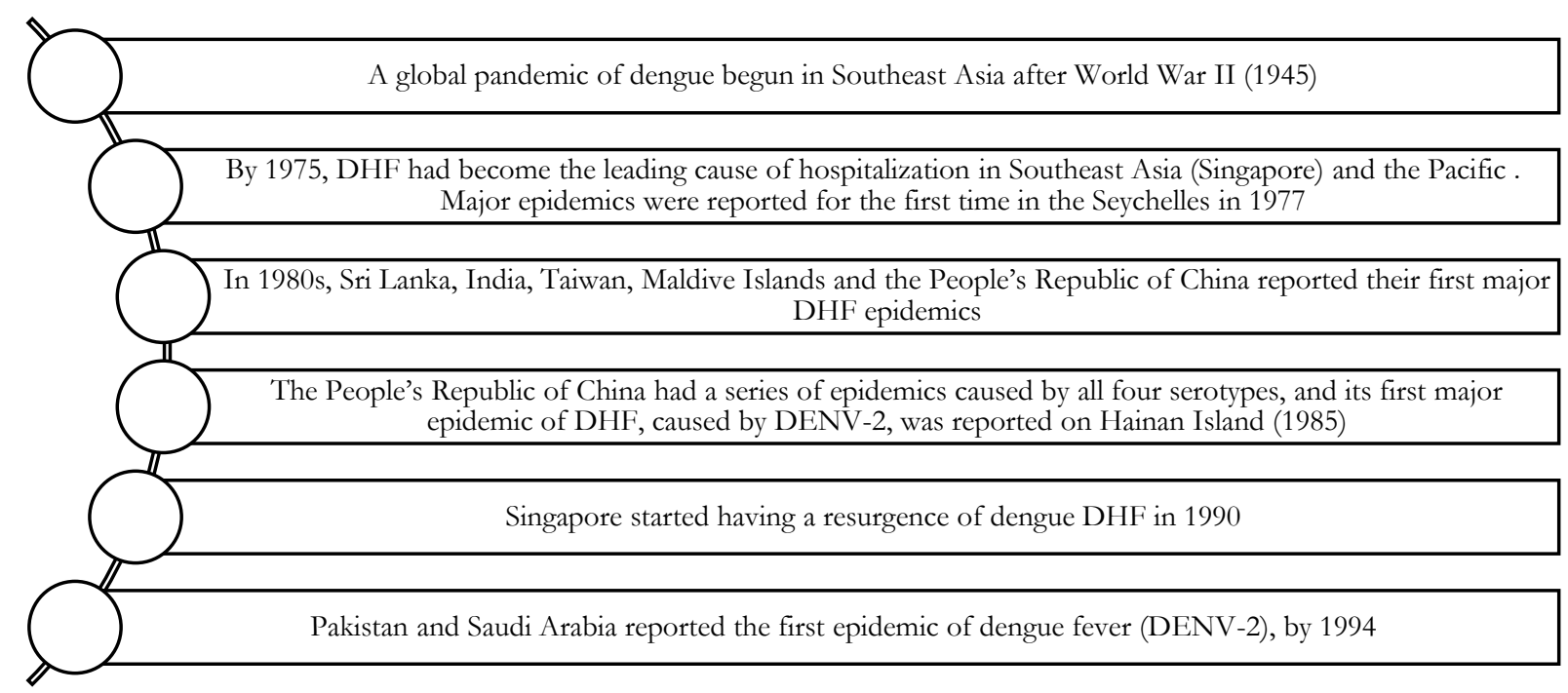

Figure 1: Summary of the expansion of dengue in Asia [2], [7], [11], [12] 


\subsection{Dengue in the American region}

The emergence of Dengue as a major public health problem has been most dramatic in the American region since when, an effort to prevent urban yellow fever by the Pan American Health Organization eradicated Aedes aegypti from most Central and South American countries in the 1950s and 1960s leading to only a few sporadic cases of epidemic Dengue in the region, concealed much on the Caribbean Islands [12]. Major epidemics caused by DENV-1 \& 4 were reported throughout the region over a 16-year period since 1977 although DENV-2 \& 3 had focal distributions in Colombia and Puerto Rico [11], [12], [16]. DENV-4 was detected during a dengue outbreak in the Amazonian city of Boa Vista in 2010, a time when Brazil was already endemic for DENV-1, DENV-2 and DENV-3 [17]. The near simultaneous occurrence of outbreaks on three continents indicates that these viruses and their mosquito vectors have had a worldwide distribution in the tropics for more than 200 years [3], [7], [9].

\section{Dengue Transmission}

Dengue viruses, unlike most arboviruses, rely on transmission by mosquito vectors that live in close association with people. The humans serve as reservoir and amplification hosts since most DENVs that cause human diseases are not zoonoses [18]. The greatest health risk of arboviral emergence is driven by extensive tropical urbanization and its colonization by the highly anthropophilic mosquito, Aedes (Stegomyia) aegypti [1], [18]. Aedes aegypti is the principal vector of Dengue fever [19]. The recent invasion into the Americas, Europe and Africa by Aedes (Stegomyia) albopictus, a secondary vector of DENV has enhanced urban transmission of these DENVs into tropical as well as temperate regions [18], [19]. DENV-3 has also been detected in other mosquito vectors like the Mansonia africana in Nigeria [20]. Even though Aedes albopictus is highly anthropophilic, it is more dependent on vegetation and associated resources associated with it thus more common in suburban and rural areas [21]. The expansion of Dengue has been attributed to the role of human transportation [22]. It should be noted that both Aedes aegypti and Aedes albopictus are aggressive daytime biters and containerinhabiting species that lay eggs in clean watercontaining receptacles in and around the home environment [21], [23]. They can also bite at night if there is sufficient artificial lighting. Aedes aegypti usually bites at the ankles of humans whereas Aedes albopictus biting peaks during early morning or late afternoon [23].

\section{Dengue Reemergence}

There are complex reasons for the dramatic reemergence of Dengue as a major global health problem although, Gubler and Clark, (2008) identified some features as summarized in Table 1 below.

Table 1: Summary of the key factors that have contributed to Dengue reemergence [5], [12].

Competing priorities or the so called "crisis mentality" within the global health system

Deteriorated public health infrastructure in low and middle income countries makes it difficult to control dengue epidemics

Paucity of mosquito control programs in areas where Dengue fever has been eradicated

Globalization by air transport and increased worldwide trade in second hand tyres has facilitated the breeding and movement of Dengue vectors

Uncontrolled urban expansion associated with the concurrent population growth have created a favourable environment for the breeding of Aedes aegypti and Aedes albopictus vectors

The inadequate capacity of most countries to implement effective mosquito control strategies especially in the realm of insecicide resistance

The inadequate capacity of effective mosquito control strategies in most dengue-endemic countries is the greatest challenge facing the world today since most adult mosquito control still relies on ultra-low-volume insecticide space sprays which are relatively ineffective in controlling Aedes mosquitoes. In addition, the so called "competing priorities" or the "crisis mentality" which emphasizes the implementation of "emergency control methods" in response to 
epidemics rather than on developing programs to prevent epidemic transmission has allowed dengue epidemics to reach or surpass the peak of transmission before it is detected [12].

\subsection{Community Involvement and Social Perspectives: A Challenge}

The stages of dengue transmission present several possibilities for behavioural intervention, through horizontal or vertical programmes, or a combination of the two, but each of these actions has different implications for the reach, adoption, effect and maintenance of the programme and, may vary according to the specific areas of application [24]. Elder and Ballenger-Browning, (2009) reviewed some of the key features that must be addressed by mosquito control and dengue control programmes to be deemed successful and they are summarized in the Figure 2 below. But unlike malaria, there is limited knowledge on social aspects of DENV epidemiology, such as understanding the degree up to which human mobility and social interactions affect exposure and transmission of DENV, and the human behavioral changes that need to occur to render a community-based intervention more effective and sustainable [3], [25]. Clearly, this is an area where multidisciplinary research is needed to fill important knowledge gaps [3].

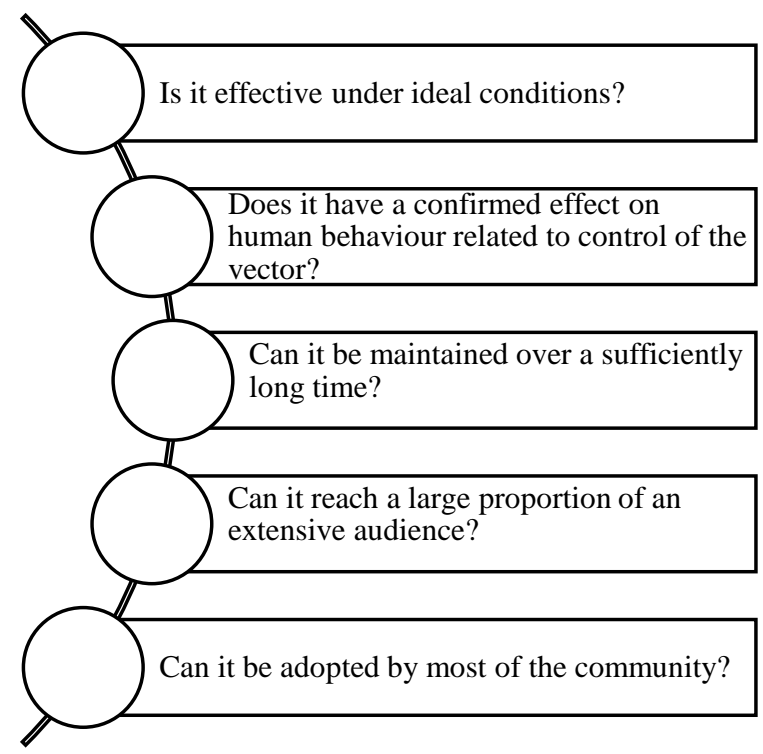

Figure 2: Important scientific questions that deem a dengue control programme successful [24], [25].
The extensive implementation of standard control actions recommended by control programmes for example in Brazil, shown to have only a slight decrease in mosquito density therefore, it is recommended that Dengue control programmes should work hand in hand with public consultation/engagement, behavioural change, intermittent information campaigns and actions that go beyond technical impositions for these programmes to be effective [17]. The increase of Dengue/DHF worldwide is everyone's responsibility therefore, increasing awareness of improved water storage practices among affected populations could drastically reduce the density of Aedes adult mosquitoes hence reducing transmission [25], [26]. Proper knowledge and information regarding dengue practices should be emphasized in dengue endemic regions [25], [27].

\subsection{Dengue Surveillance Systems: A Challenge}

The implementation of timely and effective mosquito control strategies is highly dependent on data generated by surveillance systems. The increasing levels of globalization has put travelers at a high risk of acquiring dengue infection and hence its spread to non-endemic regions however, these very travelers serve as sentinels to alert the international community of any dengue epidemics [28], [29]. This is done through a global surveillance network called the 'GeoSentinel' that monitors all travel-related illnesses [28]. As of 2012, the GeoSentinel estimated that dengue accounts for $2 \%$ of all illnesses among travelers from dengue-endemic regions and was the most frequent cause of febrile illness in travelers returning from Southeast Asia [28]. Using this kind of estimation is however dangerous since it varies according to the reporting requirements of a particular country and much affected by the non-specific clinical presentation of the disease [22], [29]. In Nigeria for example, relying on clinical diagnosis may not clearly reflect the actual dengue risk as a lot of cases go undetected [14]. Emphasizing routine diagnosis can enhance dengue virus surveillance and consequently improve patient care in West Africa. Dengue surveillance has also relied on the larval surveys 
Amos Watentena et al., Int. Ann. Sci.; Vol. 9, Issue 1, pp:132-140, 2020

and other methods monitoring the productivity of breeding containers of Dengue vectors [3]. Assessing the actual risk of human infection by DENV based on immature Aedes mosquito indices has proven difficult and it requires validation. Therefore, researchers have rather suggested the use of appropriate spatial scale of assessing the entomological risk by individual households involving the determination of relative abundance of adult vectors in relation to human serotype-specific herd immunity, climate/weather, introduction of unique viruses and mosquito-human contact [30], [31]. Also, integrating the recently developed multiplatforms application in Dengue entomological surveillance system should be emphasized across the globe to cost-effectively support the entomological unit in fieldwork during surveillance missions [32].

\subsection{Challenges with Dengue Vector Control}

The effective and sustainable control of mosquito vectors is a puzzling global health challenge yet vector control is the most widespread tool to curb dengue epidemics. The proven vector control tools recommended by the World Health Organization Vector Control Advisory Group for use in vector control programs are insufficient to meet the growing challenge of Dengue epidemics therefore, additional measures like the use of vaccines and antivirals are required [33]. The most widely adopted strategy to minimize Dengue fever has been the control of the Aedes mosquito larvae populations however, the emergence of insecticide resistance has amplified the interest in finding natural products effective against larval/adult Aedes aegypti and Aedes albopictus densities. Plant-derived compounds (botanicals and essential oils) have been recommended in vector control because they are safer and have lower toxicity to humans as compared to conventional insecticides [22], [33][35]. Also, due to strong conspecific egg-laying behavior of Aedes aegypti and Aedes albopictus populations, combining larviciding with strategies that kill mosquitoes in later stages (pupae) of their development like the insect growth regulators, will enhance vector control a phenomenon called 'density-dependent larval competition and late acting insecticide' [31], [36], [37]. Research on other methods like; the use of local gene drives, the bacterium, the eave tubes, mosquito lures, attractive toxic sugar baits (ATSBs) and night vision, has almost been completed but their application has been on a small scale [38]-[41]. Wolbacbia has shown to be an efficient novel biological control agent though transgenics still face ethical concerns [40], [42]. As scientists resort to the use genetically modified mosquitoes, the major challenge to overcome is the development of efficient genetic drive systems that spread effector genes into local vector populations [39]. Ecological models illustrating the role of mosquito control and awareness parameters on the Dengue threshold clearly outline the important role of vector control in disease prevention [43], [44]. Such models can be used to guide stakeholders in the application of pre-emptive dengue vector control thereby enhancing disease management [45]. Support from the government, public health organizations, donors, community engagement and stakeholders is critical in the development of sustainable DENV control programs [46].

\subsection{The Challenge with Diagnostic Methodologies}

Timely, efficient and accurate diagnosis of Dengue is of primary importance for outbreak control, pathogenesis, academic research, vaccine development, surveillance activities, clinical trials and clinical care [47]. Clinical care involves early detection of dengue infection, case confirmation and differential diagnosis with other infectious diseases. The most commonly used diagnostic techniques include: virus in cell culture, viral nucleic acid (molecular methods), dengue virus antigens/specific antibodies (serological tests) and hematological test (platelet and hematocrit value) [47]. For early stages, virus isolation, genome detection or antigen identification may each be used for diagnosis verses serology at acute phase. Using a combination of two or more of these techniques increases the accuracy of diagnosis. Whereas progress had been made in development of commercial test kits which claim rapid detection of anti-dengue $\operatorname{IgM}$ and $\operatorname{IgG}$ 
antibodies, most of them have been questioned or unknown because proper validation studies have not been performed and the results not yet publicly declared [47]. The NS1 antigen assay (serological diagnosis) and ELISA are the best new alternatives for antibody diagnosis due to their high degrees of specificity and sensitivity [47]. However, the clinical diagnosis in the absence of laboratory confirmation is often unreliable but the cross-sectional serologic surveys have the potential to shed light on the broader population burden of DENV without these biases though the serologic cross-reactions among antibody-based assays for Flaviviruses can limit the reliability of such studies in the absence of confirmatory testing [48]. Further studies should investigate the specificity and sensitivity of promising novel technologies.

\subsection{Global Health System: The Unresolved Hurdle}

Later in 2015, The Lancet showed how the India's health system was overrun with just 21,000 dengue cases, the worst outbreak in the previous 5 years by then. The country's national media showed state hospitals full of patients, with some sharing beds, and private hospitals refusing to provide care [49]. India's response to such acute public health emergency demonstrates the inadequate capacity of most dengue endemic countries (like Thailand, Cambodia, Bangladesh and Nigeria) in handling outbreaks and points to a healthcare system that is chronically underfunded, unregulated, and has inadequate infrastructure [49]. A similar study has confirmed a high societal and individual family burden of dengue in Cambodia [50]. The emergence and reemergence of other viruses like filoviruses (Ebola) and coronaviruses (Covid-19) has strongly made the situation worse [51]. This is most likely to overturn all the efforts which had been put in place controlling Dengue and increases competition for the healthcare.

\subsection{The Challenge with Drug Discovery}

The development of small molecule DENV inhibitors as a tool to prevent/treat Dengue faces major hurdles in combining pan-serotypic efficacy, safety, and optimal drug-like properties
[33]. Marques and Kaplan, (2015) proposed a dengue antiviral target product that could address some of these challenges although they predicted that financial return of some of these dengue drug discovery projects may not compensate for the initial investment in research and development. The successful translation from basic knowledge into effective DENV control interventions ultimately depend on the active collaboration and communication between researchers from different disciplines and on the economic support from funding agencies and institutions [3].

\subsection{Challenges with the Development of Vaccines}

There is now a better understanding of the fascinating complexities in DENV infection and transmission than before and this is beginning to yield novel and more effective control tools. Research breakthroughs over the past 20 years have yielded vaccines and myriad of a candidate vaccines although mosquito vector control remains the only and most effective mode of preventing human dengue infection [3], [4], [8]. By 2018, the first dengue vaccine, CYDtetravalent dengue vaccine (CYD-TDV) or Dengvaxia (Sanofi Pasteur, Lyon, France) was licensed in 20 countries including Mexico and Brazil though initial findings from large scale clinical trials shown good but incomplete protection against severe dengue [52]-[55]. CYDTDV is a live recombinant tetravalent dengue vaccine given as a 3 -dose series on a 0, 6 and 12 month schedule [53], [56]. Whereas progress is being made in the production of vaccines; the lack of an animal model that reproduces the disease observed in humans, the necessity of the vaccine to be effective against all 4 DENV serotypes, and the lack of an identified correlate of protection, are undermining the complexities of vaccine development [4], [57]-[59]. To address some of these challenges, humans are being used in preclinical trials which is ethically dangerous and has been underpinned by the non-existence of a licensed anti-dengue therapeutic agent that could treat a volunteer who becomes ill following challenge especially with non-protective wildtype DENV vaccination [57], [58]. In addition, 
Amos Watentena et al., Int. Ann. Sci.; Vol. 9, Issue 1, pp:132-140, 2020

Many new antiviral compounds against the NS5 protein of DENV are being investigated for example; the phosphoamidate nucleoside prodrug GS5734 also called "Remdesivir" (that is active against Ebola virus, arenaviruses, and coronaviruses) and "Favipiravir," as broadspectrum antivirals [51]. They are starting to show promising results. Predictive models have shown that weakly efficacious vaccination strategies against multiple virus serotype diversity can be counter-productive to disease control efforts [55], [60].

\subsection{Tailoring Dengue interventions in the COVID-19 response: A Challenge}

The rapid emergence and spread of COVID-19 across the world has created massive global disruptions that are impacting lives and undermining the global efforts to prevent Dengue [61]. Whereas there is remarkable work being done to address this challenge [61], it is essential not to ignore other killer diseases like malaria and Dengue/DHF. The COVID-19 pandemic could be devastating on its own but its effects can be substantially further amplified if the response undermines the provision of lifesaving services for other diseases [61], [62]. In this regard therefore, Ministries of Health and Dengue control programmes must ensure that Dengue control efforts are not hampered or neglected as they tackle the COVID-19 pandemic. The Pan-American Organization for Health reported about 560,000 new infections and 118 deaths of Dengue fever for the first two months of 2020 [62]. According to Laura Lopez in an interview with DW TV [62], the containment of DENV could work with a change in the behaviour of the population and proper direction of resources amidst COVID-19.

\section{$5 \quad$ Conclusion}

The recent reemergence of Dengue and the global expansion of Dengue vectors presents a serious global health challenge. At a time when the world is struggling with a pandemic, Dengue control may require a smart, complex and multifaceted approach outlining proper resource allocation towards prevention/control strategies, evidence-based clinical care, and mass vaccination in endemic regions, for a certain level of eradication or containment to be achieved.

\section{Declarations}

\subsection{Acknowledgments}

The authors appreciate Malaria Consortium UK for the Dr. Sylvia Meek Scholarship.

\subsection{Competing Interests}

The authors declare no competing interests.

\section{How to Cite this Article:}

A. Watentena, I. Okoye, and I. Onah, "Dengue Reemergence: The Challenges Ahead", Int. Ann. Sci., vol. 9, no. 1, pp. 132-140, Jun. 2020. doi:10.21467/ias.9.1.132140

\section{References}

[1] G. Rezza, "Aedes albopictus and the reemergence of dengue," BMC Public Health, vol. 12, no. 1, pp. 2-4, 2012.

[2] M. Kashif Zahoor et al., Dengue fever: a general perspective. IntechOpen, 2019.

[3] G. M. Vazquez-Prokopec, "Dengue control: the challenge ahead," Future Microbiol., vol. 6, no. 3, pp. 251-253, 2011.

[4] L. M. Schwartz, M. E. Halloran, A. P. Durbin, and I. M. Longini, "The dengue vaccine pipeline: implications for the future of dengue control," Vaccine, vol. 33, no. 29, pp. 3293-3298, 2015.

[5] M. S. Diamond and T. C. Pierson, "Molecular insight into dengue virus pathogenesis and its implications for disease control," Cell, vol. 162, no. 3, pp. 488-492, 2015.

[6] D. J. Gubler, "Dengue and dengue hemorrhagic fever," Clin. Microbiol. Rev., vol. 11, no. 3, pp. 480-496, 1998.

[7] D. J. Gubler, "Dengue and dengue hemorrhagic fever," in Tropical Infectious Diseases, vol. 17, no. 4, 2011, p. 813.

[8] U. C. Chaturvedi and R. Nagar, "Dengue and dengue haemorrhagic fever: Indian perspective," J. Biosci., vol. 33, no. 4, pp. 429-441, 2008

[9] W. Fan, S. Yu, and T. M. Cosgriff, "The re-emergence of dengue in China," Rev. Infect. Dis., vol. 2, no. Supl 4, 1989.

[10] T. Takasaki, "Imported dengue fever/dengue hemorrhagic fever cases in Japan," Trop. Med. Health, vol. 39, no. 4, pp. 13-15, 2011.

[11] D. J. Gubler and G. G. Clark, "Dengue hemorrhagic fever.," Emerg. Infect. Dis., vol. 1, no. 2, pp. 55-57, 1995.

[12] D. J. Gubler and G. G. Clark, "Dengue/dengue hemorrhagic fever: the emergence of a global health problem," Emerg. Infect. Dis., vol. 1, no. 2, pp. 55-57, 2008.

[13] A. H. Fagbami and A. B. Onoja, "Dengue haemorrhagic fever: an emerging disease in Nigeria, West Africa," $J$. Infect. Public Health, vol. 11, no. 6, pp. 757-762, 2018.

[14] A. B. Onoja, J. A. Adeniji, and O. D. Olaleye, "High rate of unrecognized dengue virus infection in parts of the rainforest region of Nigeria," Acta Trop., vol. 160, pp. 
39-43, 2016.

[15] F. Were, "The dengue situation in Africa," Paediatr. Int. Child Health, vol. 32, no. Sup1, pp. 18-21, 2012.

[16] A. Wilder-smith, D. J. Gubler, S. C. Weaver, T. P. Monath, D. L. Heymann, and T. W. Scott, "Epidemic arboviral diseases: priorities for research and public health," Lancet Infect. Dis., vol. 3099, no. 16, pp. 1-6, 2016.

[17] R. Maciel-de-Freitas, D. Valle, M.-F. Rafael, and V. Denise, "Challenges encountered using standard vector control measures for dengue in Boa Vista, Brazil," Bull. World Health Organ., vol. 92, no. 9, pp. 685-689, 2014.

[18] S. C. Weaver and W. K. Reisen, "Present and future arboviral threats," Antiviral Res., vol. 85, no. 2, pp. 328345,2010

[19] L. Braack, A. P. Gouveia De Almeida, A. J. Cornel, R. Swanepoel, and C. De Jager, "Mosquito-borne arboviruses of African origin: Review of key viruses and vectors," Parasites and Vectors, vol. 11, no. 1, 2018.

[20] C. Isaac and E. J. Agwu, "Mosquito infection with dengue and yellow fever in Bayelsa and Benue States, Nigeria,” Int. J. Infect. Dis., vol. 45, pp. 73-74, 2016.

[21] R. Barrera, "Considerations for disrupting dengue virus transmission; ecology of Aedes aegypti and current (nongenetic) methods of control," in Genetic Control of Malaria and Dengue, Elsevier Inc., 2016, pp. 103-124.

[22] P. Kittayapong, "Malaria and dengue vector biology and control in Southeast Asia," in Bridging Laboratory and Field Research for Genetic Control of Disease Vectors, 2007, pp. 111-127.

[23] S. C. Arya and N. Agarwal, "Apropos: An update on the incidence of dengue gaining strength in Saudi Arabia and current control approaches for its vector mosquito," Parasites and Vectors, vol. 7, no. 1, pp. 1-2, 2014.

[24] J. P. Elder and K. Ballenger-Browning, "Community involvement in dengue vector control," $B M J$, vol. 338, no. b2210, pp. 1453-1455, 2009.

[25] M. F. F. Said, H. Abdullah, and N. Abdul Ghafar, "Dengue prevention practices among community in dengue hotspot area," Int. J. Community Med. Public Heal., vol. 5, no. 11, p. 4664, 2018.

[26] T. Mariappan, "Dengue transmission," Environ. Health Perspect., vol. 117, no. 2, pp. 56-57, 2009.

[27] V. Balan, "Meeting the dengue fever challenge," Biol. Syst. Open Access, vol. 04, no. 01, p. 135, 2015.

[28] A. Wilder-smith, "Dengue infections in travellers," Paediatr. Int. Child Health, vol. 32, no. Sup 1, pp. 2832, 2012, doi: 10.1179/2046904712Z.00000000050.

[29] M. Izadi, A. Is'haqi, M. A. Is'haqi, N. J. Jafari, F. Rahamaty, and A. Banki, "An overview of travelassociated central nervous system infectious diseases: risk assessment, general considerations and future directions," Asian Pac. J. Trop. Biomed., vol. 4, no. 8, pp. 589-596, 2014.

[30] T. W. Scott and A. C. Morrison, "Vector dynamics and dengue prevention," in Dengue Virus, Current Topics in Microbiology and Immunology, vol. 338, no. 1, A. L. Rothman, Ed. Springer-Verlag Berlin Heidelberg 2010, 2009, pp. 115-128.

[31] K. T. Wai et al., "Estimating dengue vector abundance in the wet and dry season: implications for targeted vector control in urban and peri-urban Asia," Pathog. Glob. Health, vol. 106, no. 8, pp. 436-445, 2012.

[32] N. Ibrahim and T. W. Quan, "The development of multiplatforms application for dengue-entomological surveillance system," IEEE, 2017.
[33] A. M. Marques and M. A. C. Kaplan, "Active metabolites of the genus Piper against Aedes aegypti: Natural alternative sources for dengue vector control," Univ. Sci., vol. 20, no. 1, pp. 61-82, 2015.

[34] A. R. Ishak, N. C. Dom, H. Hussain, and N. H. Sabri, "Biolarvacidal potential of Ipomoea cairica extracts against key dengue vectors," Procedia - Soc. Behav. Sci., vol. 153, pp. 180-188, 2014.

[35] H. A. A. Khan and W. Akram, "Citrus-based essential oils could be used for dengue vector mosquitoes control," Asian Pac. J. Trop. Med., vol. 6, no. 6, p. 504, 2013.

[36] J. Wong, S. T. Stoddard, H. Astete, A. C. Morrison, and T. W. Scott, "Oviposition site selection by the dengue vector Aedes aegypti and its implications for dengue control," PLoS Negl. Trop. Dis., vol. 5, no. 4, p. e1015, 2011.

[37] M. de B. Arduino, "Assessment of Aedes aegypti pupal productivity during the dengue vector control program in a costal urban centre of São Paulo State, Brazil," $J$. Insects, vol. 2014, pp. 1-9, 2014.

[38] F. O. Okumu and S. J. Moore, "Combining indoor residual spraying and insecticide-treated nets for malaria control in Africa: a review of possible outcomes and an outline of suggestions for the future," Malar. J., vol. 10, no. 208, pp. 1-13, 2011.

[39] K. E. Olson and A. W. E. Franz, "Controlling dengue virus transmission in the field with genetically modified mosquitoes," in Advances in Human Vector Control, vol. 1014, J. Clark, Ed. Washington DC: American Chemical Society, 2009, pp. 123-141.

[40] L. A. Moreira, "Wolbachia as part of a dengue control strategy - challenge Brazil," in 2016 XXV International Congress of Entomology, 2016.

[41] A. Watentena and I. C. Okoye, "The untapped potential of mosquito lures for malaria vector surveillance and mass trapping of mosquitoes: a review," Int. J. Mosq. Res., vol. 6, no. 1, pp. 132-137, 2019.

[42] A. J. Richardson and C. R. Williams, "Inter-population mating success in Australian dengue vector mosquitoes: effects of laboratory colonization and implications for the spread of transgenics," J. Vector Ecol., vol. 38, no. 1, pp. 111-119, 2013.

[43] A. Mishra and S. Gakkhar, "The effects of awareness and vector control on two strains dengue dynamics," Appl. Math. Comput., vol. 246, pp. 159-167, 2014.

[44] R. E. Sinden, "The challenge of disrupting vectorial capacity," in Genetic Control of Malaria and Dengue, Elsevier Inc., 2016, pp. 125-138.

[45] A. H. Azil, S. A. Long, S. A. Ritchie, and C. R. Williams, "The development of predictive tools for pre-emptive dengue vector control: a study of Aedes aegypti abundance and meteorological variables in North Queensland, Australia," Trop. Med. Int. Heal., vol. 15, no. 10, pp. 1190-1197, 2010.

[46] M. A. McArthu, M. B. Sztein, and R. Edelman, "Prospects for controlling dengue spread: vaccines and vector control," in Clinical Insights: Dengue: Transmission, Diagnosis \& Surveillance, Future Medicine Ltd, 2014, pp. 103-119.

[47] D. M. Khan, "Diagnostic methodologies for the dengue viral infection," Int. J. Heal. Allied Sci., vol. 5, no. 2, p. 133, 2016.

[48] A. Salem Bin Ghouth, "Dengue in the WHO Eastern Mediterranean Region: challenges to understand its epidemiology," Heal. Prim. Care, vol. 2, no. 2, pp. 1-2, 2018 . 
[49] The Lancet, "Dengue challenges India's health system," Lancet, vol. 386, no. 10000, p. 1212, 2015.

[50] J. Beauté and S. Vong, "Cost and disease burden of Dengue in Cambodia," BMC Public Health, vol. 10, no. 1, pp. 2-7, 2010, doi: 10.1186/1471-2458-10-521.

[51] A. Badolo et al., "Third Tofo advanced study week on emerging and re-emerging viruses, 2018," Antiviral Res., vol. 162, pp. 142-150, 2019.

[52] K. K. Ariën and A. Wilder-Smith, "Dengue vaccine: reliably determining previous exposure," Lancet Glob. Heal., vol. 6, no. 8, pp. e830-e831, 2018.

[53] M. Recker, K. Vannice, J. Hombach, M. Jit, and C. P. Simmons, "Assessing dengue vaccination impact: model challenges and future directions," Vaccine, vol. 34, no. 38, pp. 4461-4465, 2016.

[54] M. L. Robinson and A. P. Durbin, "Dengue vaccines: implications for dengue control," Curr. Opin. Infect. Dis., vol. 30, no. 5, pp. 449-454, 2017.

[55] S. Polwiang, "The effectiveness of dengue vaccine and vector control: Model study," KMUTNB Int. J. Appl. Sci. Technol., vol. 11, no. 3, pp. 225-232, 2018.

[56] G. N. Lucas, "A fresh look at dengue vaccines," Sri Lanka Journalof Child Heal., vol. 45, no. 3, pp. 149-150, 2016.

[57] A. P. Durbin and S. S. Whitehead, "The dengue human challenge model: has the time come to accept this challenge?," J. Infect. Dis., vol. 207, no. 5, pp. 697-699, 2013.

[58] R. C. Christofferson and C. N. Mores, "A role for vector control in dengue vaccine programs," Vaccine, vol. 33, no. 50, pp. 7069-7074, 2015.

[59] M. S. Diamond, E. Harris, and F. A. Ennis, "Defeating dengue: a challenge for a vaccine," Nat. Med., vol. 18, no. 11, pp. 1622-1623, 2012.

[60] R. W. S. Hendron and M. B. Bonsall, "The interplay of vaccination and vector control on small dengue networks," J. Theor. Biol., vol. 407, pp. 349-361, 2016.

[61] Global Malaria Programme, "Tailoring malaria interventions in the COVID-19 response," World Health Organization, Geneva, 2020.

[62] DW Television, "The coronavirus spreads at a fractic pace between Tijuana and Tierra del Fuego," Latin America, 2020.
Publish your books with AIJR publisher$\checkmark \quad$ Publish with ISBN and DOI.

$\checkmark \quad$ Publish Thesis/Dissertation as Monograph.

$\checkmark \quad$ Publish Book Monograph.

$\checkmark \quad$ Publish Edited Volume/ Book.

$\checkmark \quad$ Publish Conference Proceedings

$\checkmark \quad$ Retain full copyright of your books.

Submit your manuscript at books.aijr.org

Publish your research article in AIJR journals$\checkmark \quad$ Online Submission and Tracking

$\checkmark$ Peer-Reviewed

$\checkmark \quad$ Rapid decision

$\checkmark \quad$ Immediate Publication after acceptance

$\checkmark \quad$ Articles freely available online Retain full copyright of your article.

Submit your article at journals.aijr.in 